\title{
Polyakov Loops for the ABJ Theory
}

\author{
Mir Faizal ${ }^{1}$ and Tsou Sheung Tsun ${ }^{2}$ \\ ${ }^{1}$ Department of Physics and Astronomy, \\ University of Waterloo, Waterloo, \\ Ontario N2L 3G1, Canada \\ ${ }^{2}$ Mathematical Institute, University of Oxford \\ Oxford OX1 3LB, United Kingdom
}

\begin{abstract}
In this paper we will first construct Polyakov loops for the ABJ theory. Then we will construct the connection and curvature in this loop space. We will also analyse certain generalization of Polyakov loops and apply them to the ABJ theory. Finally, we will use this formalism for analysing topological defects like monopoles in the ABJ theory.
\end{abstract}

\section{Introduction}

According to the $A d S / C F T$ correspondence the eleven dimensional supergravity on $A d S_{4} \times S_{7}$ is dual to a superconformal field theory describing multiple M2-branes. This superconformal field theory has to have $\mathcal{N}=8$ supersymmetry. This is because apart from a constant closed 7-form on $S^{7}, A d S_{4} \times S^{7} \sim$ $[S O(2,3) / S O(1,3)] \times[S O(8) / S O(7)] \subset O S p(8 \mid 4) /[S O(1,3) \times S O(7)] . \quad$ The $O S p(8 \mid 4)$ gets realized as $\mathcal{N}=8$ supersymmetry of the dual superconformal field theory. The transverse coordinates of the M2-branes give rise to eight gauge valued scalar fields. Apart from these eight gauge valued scalar fields, this theory also has sixteen physical fermions. The gauge fields of this theory do not have any on-shell degrees of freedom. A theory called the BLG theory satisfies these properties 1, 2, 3, 4, 5. However, the gauge symmetry of the BLG theory is based on a Lie 3-algebra and the only known example of a Lie 3-algebra is $S O(4) \sim S U(2) \times S U(2)$. So, the BLG theory can only describe two M2-branes.

It has been possible to generalize the BLG theory to a superconformal field theory describing any number of M2-branes on $A d S_{4} \times S_{7} / Z_{k}, 6,7,8,9$. This theory called the ABJM theory only has $\mathcal{N}=6$ supersymmetry and $S O(6)$ $R$-symmetry. However, as it considers with the BLG theory for two M2-branes, it is expected that its supersymmetry would get enhanced to full $\mathcal{N}=8$ supersymmetry. In fact, the supersymmetry for the ABJM can gets enhanced to $\mathcal{N}=8$ supersymmetry for Chern-Simons levels, $k=1,2$, by the use of monopole operators 10, 11, 12, 13. In the ABJM theory the matter fields are in the bi-fundamental representation of the gauge group $U(N) \times U(N)$ and the double gauge fields are in the adjoint representation. A further generalization of the ABJM theory to a theory describing fractional M2-branes has been made 
14, 15, 16, 17, 18. This theory is called the ABJ theory and in it the gauge fields are described by the gauge group $U(M) \times U(N)$ with $M \neq N$ [19, 20]. The matter fields are again in the bi-fundamental representation of this gauge group and the double gauge fields are in the adjoint representation.

Wilson loops for the ABJ theory have been studied and they are given by semi-classical string surfaces in the dual string theory picture 21, 22. The most symmetric string of this kind preserves half the supersymmetry. The dual field theory operator to it has also been constructed using a superconnection 23. In this superconnection, the scalar fields occur in bi-linears combinations and the fermions appear linearly. Thus, the fermions transform in the bi-fundamental representation and appear in the off-diagonal block. The bi-linear product of the scalars transforms in the adjoint representations. So, the scalars appear in the diagonal blocks along with the gauge fields. The fermions couple to Grassmann even quantities and thus the off-diagonal blocks contain Grassman odd quantities. It may be noted that Wilsons loops which preserve $1 / 6$ of the total supersymmetry have also been studied 24, 25. In fact, a matrix model corresponding to the vacuum expectation value for the $1 / 6$ BPS Wilsons loop has been constructed [26].

In this paper we introduce Polyakov loops as the variables to be used. In mathematical language these are the holonomies of closed loops in space-time, and they are sometimes also called Dirac phase factors in the physics literature. Although they are defined via parametrized loops in space-time, they are independent of the parametrization chosen. They are therefore gauge group-valued functions of the infinite-dimensional loop space. The main difference between a Polyakov loop and a Wilson loop is that in the Wilson loop a trace is taken and no such such trace is taken in the Polyakov loop 27. In this paper we will study the Polyakov loops for the ABJ theory. Polyakov loops have been used for deriving a duality in non-abelian gauge theories [28, 29. This duality has been used for analysing the 't Hooft's order-disorder parameters 30. A Dualized Standard Model has also been constructed using this duality [31, 32. In this model three generations of fermions are produced by the breaking of a dual color $S U(3)$ symmetry [33, 34. The resulting scheme give a method for calculating a fermion mass hierarchy along with the mixing parameters of the Standard Model fermions 35, 36. Dual Feynman Rules for Yang-Mills theories with a monopole have also been analysed using Polyakov loops 37. Polyakov loops for supersymmetric gauge theories in $\mathcal{N}=1$ superspace have also been discussed [38.

It is possible to define a Polyakov connection on loop space which measures the change in phase as one moves from one point in the loop space to a neighboring point. It is also possible to construct a curvature tensor using this connection [39, 40. This curvature is proportional to the Bianchi identities and thus vanishes when the Bianchi identities are satisfied [1]. As in the presence of a monopoles, Bianchi identities are not satisfied, so this curvature only gets a non-zero value when a monopole is present. Furthermore, it is possible to define a loop in the loop space which covers a surface in spacetime. This loop in the loop space can be used as a measure for the non-abelian monopole charge. These results are know to hold for ordinary Yang-Mills theories. We shall derive them for the ABJ theory. We shall also generalize some of the previously known results. So, we shall obtain a curvature and connection in the space of loop of loops and use them for analysing topological defects in the loop space. 


\section{Polyakov Loops}

As the ABJ theory is a Chern-Simons-Matter theory with the gauge group $U(N) \times U(M)$, so, we will denote the gauge fields corresponding to $U(N)$ by $A_{\mu}$ and the gauge fields corresponding to $U(M)$ by $A_{\mu}^{\prime}$. These gauge fields are coupled to complex scalar fields $C_{I}$ and their complex conjugates $\bar{C}^{I}$, where $I=1 . .4$ is an $S U(4)_{R}$ index. They are also coupled to fermions $\phi_{I}^{a}$ and $\bar{\phi}_{\underline{a}}^{I}$, where $a= \pm$ is a spinor index. It may be noted that the matter fields $C_{I}, \bar{\phi}_{a}^{I}$ transforms under $(N, \bar{M})$ and the matter fields $\bar{C}^{I}, \phi_{I}^{a}$ transforms under $(\bar{N}, M)$ representations of the gauge group $U(N) \times U(M)$. We choose a notation such that $\bar{C}^{I} C_{I}$ and $\phi_{I}^{a} \bar{\phi}_{a}^{I}$ are in the adjoint representation of $U(N)$ and $C_{I} \bar{C}^{I}$ and $\bar{\phi}_{I}^{a} \phi_{a}^{I}$ is in the adjoint representation of $U(M)$. These fields for the ABJ theory transform under a superconformal transformations as follows

$$
\begin{aligned}
\delta A_{\mu}= & \frac{4 \pi i}{k} \bar{\Theta}^{I J \alpha}\left(\gamma_{\mu}\right)_{\alpha}^{\beta}\left(C_{I} \Psi_{J \beta}+\frac{1}{2} \epsilon_{I J K L} \bar{\Psi}_{\beta}^{K} \bar{C}^{L}\right), \\
\delta A_{\mu}^{\prime}= & \frac{4 \pi i}{k} \bar{\Theta}^{I J \alpha}\left(\gamma_{\mu}\right)_{\alpha}^{\beta}\left(\Psi_{J \beta} C_{I}+\frac{1}{2} \epsilon_{I J K L} \bar{C}^{L} \bar{\Psi}_{\beta}^{K}\right), \\
\delta C_{K}= & \bar{\Theta}^{I J \alpha} \epsilon_{I J K L} \bar{\Psi}_{\alpha}^{L}, \\
\delta \bar{C}^{K}= & 2 \bar{\Theta}^{K L \alpha} \Psi_{L \alpha}, \\
\delta \Psi_{K}^{\beta}= & -i \bar{\epsilon}^{I L \beta} \epsilon_{I L K J} \bar{C}^{J}-i \bar{\Theta}^{I J \alpha} \epsilon_{I J K L}\left(\gamma^{\mu}\right)_{\alpha}^{\beta} D_{\mu} \bar{C}^{L} \\
& +\frac{2 \pi i}{k} \bar{\Theta}^{I J \beta} \epsilon_{I J K L}\left(\bar{C}^{L} C_{P} \bar{C}^{P}-\bar{C}^{P} C_{P} \bar{C}^{L}\right) \\
& +\frac{4 \pi i}{k} \bar{\Theta}^{I J \beta} \epsilon_{I J M L} \bar{C}^{M} C_{K} \bar{C}^{L}, \\
\delta \bar{\Psi}_{\beta}^{K}= & -2 i \bar{\Theta}^{K L \alpha}\left(\gamma^{\mu}\right)_{\alpha \beta} D_{\mu} C_{L}-2 i \bar{\epsilon}_{\beta}^{K L} C_{L} \\
& -\frac{4 \pi i}{k} \bar{\Theta}_{\beta}^{K L}\left(C_{L} \bar{C}^{M} C_{M}-C_{M} \bar{C}^{M} C_{L}\right) \\
& -\frac{8 \pi i}{k} \bar{\Theta}_{\beta}^{I J} C_{I} \bar{C}^{K} C_{J} .
\end{aligned}
$$

As we want to study Polyakov loops for the ABJ theory, we consider all the loops passing through some fixed point in spacetime,

$$
C:\left\{\xi^{\mu}(s): s=0 \rightarrow 2 \pi, \xi^{\mu}(0)=\xi^{\mu}(2 \pi)\right\},
$$

where $\xi^{\mu}(s)$ represents the spacetime coordinates of all points on the loop. We also define $\dot{\xi}^{\mu}=d \xi^{\mu} / d s$, and $|\dot{\xi}|=\sqrt{\eta_{\mu \nu} \dot{\xi}^{\mu} \dot{\xi}^{\nu}}$. Even though, the gauge group of the ABJ theory is $U(N) \times U(M)$, we will embed it into a superconnection $\mathcal{A}$ belonging to $U(N \mid M)$ 23. Scalar fields occur as bi-linears because in three dimensions the dimension of scalar fields is $1 / 2$. As the bi-linear combinations of scalar fields is in adjoint representation, they occur with the gauge fields in the diagonal blocks. Furthermore, as the dimensions of the fermions in three dimensions is 1, they appear linearly. As the fermions transform under bi-fundamental representation, they are placed off-diagonally. We define $M_{J}^{I}, M_{J}^{I}, \eta_{I}^{a}, \bar{\eta}_{a}^{I}$ as the parameters in the theory which parameterize the local couplings. Even though $\eta_{I}^{a}, \bar{\eta}_{a}^{I}$ transform under a spinor representation of the Lorentz group, they are taken to be Grassmann even quantities. This is because by taking them to 
be Grassmann even quantities the off-diagonal entries become Grassmann odd quantities. So, the superconnection for $U(N \mid M)$ can be written as,

$$
\mathcal{A}[\xi]=\left(\begin{array}{cc}
\mathcal{A}_{11}[\xi] & \mathcal{A}_{12}[\xi] \\
\mathcal{A}_{21}[\xi] & \mathcal{A}_{22}[\xi]
\end{array}\right),
$$

where

$$
\begin{aligned}
& \mathcal{A}_{11}[\xi]=A_{\mu} \dot{\xi}^{\mu}+\frac{2 \pi}{k}|\dot{\xi}| M_{J}^{I} C_{I} \bar{C}^{J}, \\
& \mathcal{A}_{12}[\xi]=\sqrt{\frac{2 \pi}{k}}|\dot{\xi}| \eta_{I}^{a} \bar{\phi}_{a}^{I}, \\
& \mathcal{A}_{21}[\xi]=\sqrt{\frac{2 \pi}{k}}|\dot{\xi}| \phi_{I}^{a} \bar{\eta}_{a}^{I}, \\
& \mathcal{A}_{22}[\xi]=A_{\mu}^{\prime} \dot{\xi}^{\mu}+\frac{2 \pi}{k}|\dot{\xi}| M^{\prime}{ }_{J}^{I} \bar{C}^{J} C_{I} .
\end{aligned}
$$

So, we can write the field strength for this theory as $\mathcal{F}[\xi]=d \mathcal{A}[\xi]+\mathcal{A}[\xi] \wedge \mathcal{A}[\xi]$. The Bianchi identity can now be written as $(d+A[\xi] \wedge) \mathcal{F}[\xi]=0$.

In the dual string picture, the operators describing semi-classical string surfaces have a local $U(1) \times S U(3) R$-symmetry. So, the $R$-symmetry of the couplings can be described by a vector $n_{I}$ and its complex conjugate $\bar{n}_{I}[42$. These specify the local embedding of of $S U(3)$ subgroup into $S U(4)$. They satisfy $n_{I} \bar{n}^{I}=1$. Now we have, $\eta_{I}^{\alpha}=n_{I} \eta^{\alpha}, \bar{\eta}_{\alpha}^{I}=\bar{n}^{I} \bar{\eta}_{\alpha}, M_{J}^{I}=p_{1} \delta_{J}^{I}-2 p_{2} n_{J} \bar{n}^{I}, M_{J}^{\prime}=$ $q_{1} \delta_{J}^{I}-2 q_{2} n_{J} \bar{n}^{I}$. The Eigenvalues of the $M_{J}^{I}$ and $M^{\prime I}{ }_{J}^{I}$ are controlled the functions $p_{i}$ and $q_{i}$. The condition that the supersymmetric variation of the superconnection vanishes is too strong and it does not yield any solution for the couplings. So, it is replaced by the requirement that the supersymmetry variation of the superconnection is equal to the covariant derivative generated from it.

The spinor couplings are given by $\delta_{\alpha}^{\beta}=\left(\eta^{\beta} \bar{\eta}_{\alpha}-\eta_{\alpha} \bar{\eta}^{\beta}\right) / 2 i$ and $\left(\dot{x}^{\mu} \gamma_{\mu}\right)_{\alpha}^{\beta}=$ $\ell|\dot{x}|\left(\eta^{\beta} \bar{\eta}_{\alpha}+\eta_{\alpha} \bar{\eta}^{\beta}\right) / 2 i$. Furthermore, we have $M_{J}^{I}=M_{J}^{\prime I}=\ell\left(\delta_{K}^{J}-2 n_{K} \bar{n}^{J}\right)$. Here $\ell= \pm 1$ and specifies the eigenvalues of these matrices. Now $\epsilon_{I J K L}\left(\eta \bar{\Theta}^{I J}\right) \bar{n}^{K}=0$ and $n_{I}\left(\bar{\eta} \bar{\Theta}^{I J}\right)=0$ are the constraints on $\bar{\Theta}^{I J}$. Apart from these constraints, it also satisfied $\bar{\Theta}^{I J}(d / d s) \bar{\eta}^{K} \epsilon_{I J K L}=0$ and $\bar{\Theta}^{I J}(d / d s) \eta_{I}=0$. These conditions are local and a conformal Killing spinor which satisfies these constraints has to be constructed for obtaining a supersymmetric Polyakov loop. If $\bar{\theta}^{I J}$ and $\bar{\epsilon}^{I J}$ are constant spinors, then we can write, $\bar{\Theta}^{I J}=\bar{\theta}^{I J}-\left(\gamma^{\mu} \xi_{\mu}\right) \bar{\epsilon}^{I J}$.

Recall that the Polyakov loop 27] by the very definition is an element of the gauge group. Now the Polyakov loop variables for the ABJ will be given by

$$
\begin{aligned}
\phi[\xi] & =\left(\begin{array}{ll}
\phi_{11}[\xi] & \phi_{12}[\xi] \\
\phi_{21}[\xi] & \phi_{22}[\xi]
\end{array}\right) \\
& =P_{s} \exp \int d s\left(\begin{array}{ll}
\mathcal{A}_{11}[\xi] & \mathcal{A}_{12}[\xi] \\
\mathcal{A}_{21}[\xi] & \mathcal{A}_{22}[\xi]
\end{array}\right) .
\end{aligned}
$$

Here the ordering from right to left in $s$ is denoted by $P_{s}$. It may be noted that $\phi[\xi]$ depends only on the loop $C$ in spacetime and not in the manner in which it is parametrized. If we introduce a new parameter say, $s^{\prime}=f(s)$, it will only give a change in the variable of integration and not its value. So, at first sight it might appear better to define the loops as equivalence classes 
of the function $\xi(s)$, equivalent under reparametrization. But then it would be very difficult to define differentiation and integration in this quotient space of equivalence classes, and hence we will retain the original definition of the parametrized loops.

\section{Connection and Curvature}

In this section we will construct a connection and a curvature for the loop space. Strictly speaking, these do not have the exact geometric meanings of the corresponding concepts in fiber bundles [43, but the formulae obtained below make sense in the context of loop space variables and we shall continue to use these terms with this understanding. We will first obtain a connection in the loop space and relate it to the field strength in spacetime. Then, we will construct a covariant derivative using this connection. Finally, we will construct the curvature in the loop space from the commutator of these covariant derivatives. Now we first construct the connection in the loop space from $\phi[\xi]$, by taking its logarithmic derivative. As $\phi[\xi]$ is an element of the gauge group, its logarithmic derivative will be an element of the Lie algebra corresponding to that gauge group. So, we define the connection generated from $\phi[\xi]$ as follows,

$$
F_{\mu}[\xi \mid s]=\left(\begin{array}{ll}
F_{\mu}[\xi \mid s]_{11} & F_{\mu}[\xi \mid s]_{12} \\
F_{\mu}[\xi \mid s]_{21} & F_{\mu}[\xi \mid s]_{22}
\end{array}\right)
$$

where

$$
F_{\mu}[\xi \mid s]=i \phi^{-1}[\xi] \frac{\delta}{\delta \xi^{\mu}(s)} \phi[\xi] .
$$

As $F_{\mu}[\xi \mid s]$ represents the change in $\phi[\xi]$ as one moves from one point in the loop space to its neighboring point, it can be regarded as a connection in parametrized loop space. In calculations it is sometimes useful to define further $\phi\left[\xi\left(s_{1}, s_{2}\right)\right]$ as a parallel transport from a point $\xi\left(s_{1}\right)$ to a point $\xi\left(s_{1}\right)$ along the curve $C$,

$$
\begin{aligned}
\phi\left[\xi\left(s_{1}, s_{2}\right)\right] & =\left(\begin{array}{ll}
\phi_{11}\left[\xi\left(s_{1}, s_{2}\right)\right] & \phi_{12}\left[\xi\left(s_{1}, s_{2}\right)\right] \\
\phi_{21}\left[\xi\left(s_{1}, s_{2}\right)\right] & \phi_{22}\left[\xi\left(s_{1}, s_{2}\right)\right]
\end{array}\right) \\
& =P_{s} \exp \int_{s_{1}}^{s_{2}} d s\left(\begin{array}{ll}
\mathcal{A}_{11}[\xi(s)] & \mathcal{A}_{12}[\xi(s)] \\
\mathcal{A}_{21}[\xi(s)] & \mathcal{A}_{22}[\xi(s)]
\end{array}\right) .
\end{aligned}
$$

Now using $\phi\left[\xi\left(s_{1}, s_{2}\right)\right]$, we can move from a fixed point another point say, $s$, and then take a detour and travel backwards along the same path to the original point. In doing this the phase factor generated in going from the original point to $s$, exactly cancels the phase factor generated in going back from $s$ to the original point. However, the phase factor while transporting around the infinitesimal circuit at $s$ does have a finite contribution that does not cancel. In fact, this contribution is proportional to the field strength $\mathcal{F}$. Thus, $F_{\mu}[\xi \mid s]$ is proportional to $\phi^{-1}[\xi(s, 0)] \mathcal{F}[\xi(s)] \phi[\xi(s, 0)]$. In fact, it is already know that in Yang-Mills theories the connection in loop space is proportional to the field strength in spacetime [39]. We have observed here that this also hold for the superconnection of the ABJ theory.

In the loop space $F_{\mu}[\xi]$ acts like a connection. The natural quantity to construct from this connection is the curvature of the loop space. Now we can 
define a covariant derivative in the loop space as follows,

$$
\nabla_{\mu}[\xi(s)]=\frac{\delta}{\delta \xi^{\mu}(s)}+i F_{\mu}[\xi \mid s] .
$$

The curvature $-i G_{\mu \nu}\left[\xi, s_{1}, s_{2}\right]$ of the loop space can be defined by taking a commutator of these two covariant derivatives, $\left[\nabla_{\mu}\left[\xi\left(s_{1}\right)\right], \nabla_{\nu}\left[\xi\left(s_{2}\right)\right]\right]$. Thus, we can write

$$
\begin{aligned}
G_{\mu \nu}\left[\xi\left(s_{1}, s_{2}\right)\right]= & \frac{\delta}{\delta \xi^{\mu}\left(s_{2}\right)} F_{\nu}\left[\xi \mid s_{1}\right]-\frac{\delta}{\delta \xi^{\nu}\left(s_{1}\right)} F_{\mu}\left[\xi \mid s_{2}\right] \\
& +i\left[F_{\mu}\left[\xi \mid s_{1}\right], F_{\mu}\left[\xi \mid s_{2}\right]\right] .
\end{aligned}
$$

The gauge transformations in loop space can be denoted by given by $u=$ $\exp i \Lambda[\xi]$. The connection $F_{\mu}[\xi \mid s]$ transforms under these gauge transformations as $F_{\mu}[\xi \mid s]=i u \nabla_{\mu}[\xi(s)] u^{-1}$ and $G_{\mu \nu}\left[\xi\left(s_{1}, s_{2}\right)\right]$ transforms under these gauge transformations as $u G_{\mu \nu}\left[\xi\left(s_{1}, s_{2}\right)\right] u^{-1}$.

Now if we first travel from point say $s_{1}$ along a certain direction till a point say $s_{2}$. After that we travel along another direction at $s_{1}$, then we travel along the first direction and finally again travel along the direction we traveled from $s_{2}$, to get to $s_{1}$. In doing so we completed a full circuit and the total change in phase generated in the process is represented is proportional to $\phi^{-1}\left[\xi\left(s_{1}, 0\right)\right] \nabla^{*} \mathcal{F}\left[\xi\left(s_{1}\right)\right] \phi\left[\xi\left(s_{1}, 0\right)\right] \delta\left(s_{1}-s_{2}\right)$ 41. We have observed here that this also hold for the superconnection of the ABJ theory. Now this is also the value of by $-i G_{\mu \nu}\left[\xi\left(s_{1}, s_{2}\right)\right] \delta \xi^{\mu}\left(s_{1}\right) \xi^{\nu}\left(s_{2}\right)$. Hence, the curvature is proportional to $\phi^{-1}\left[\xi\left(s_{1}, 0\right)\right] \nabla^{*} \mathcal{F}\left[\xi\left(s_{1}\right)\right] \phi\left[\xi\left(s_{1}, 0\right)\right] \delta\left(s_{1}-s_{2}\right)$. Thus, if the Bianchi identity is satisfied $\nabla^{*} \mathcal{F}\left[\xi\left(s_{1}\right)\right]=0$, this curvature vanishes $G_{\mu \nu}\left[\xi\left(s_{1}, s_{2}\right)\right]=0$. However, in presence of a monopole, Bianchi identity is not satisfied and thus this curvature does not vanish.

It may be noted that $G_{\mu \nu}\left[\xi\left(s_{1}, s_{2}\right)\right]$ satisfies a functional Bianchi identity even in presence of a monopole. To derive this functional Bianchi identity, we first define $\nabla_{\mu}\left[\xi\left(s_{1}\right)\right]^{*}\left[\nabla_{\nu}\left[\xi\left(s_{2}\right)\right], \nabla_{\rho}\left[\xi\left(s_{3}\right)\right]\right]$ as follows,

$$
\begin{aligned}
& \nabla_{\mu}\left[\xi\left(s_{1}\right)\right]^{*}\left[\nabla_{\nu}\left[\xi\left(s_{2}\right)\right], \nabla_{\rho}\left[\xi\left(s_{3}\right)\right]\right] \\
= & \nabla_{\rho}\left[\xi\left(s_{3}\right)\right]\left[\nabla_{\mu}\left[\xi\left(s_{1}\right)\right], \nabla_{\nu}\left[\xi\left(s_{2}\right)\right]\right] \\
& +\nabla_{\nu}\left[\xi\left(s_{2}\right)\right]\left[\nabla_{\rho}\left[\xi\left(s_{3}\right)\right], \nabla_{\mu}\left[\xi\left(s_{1}\right)\right]\right] \\
& +\nabla_{\mu}\left[\xi\left(s_{1}\right)\right]\left[\nabla_{\nu}\left[\xi\left(s_{2}\right)\right], \nabla_{\rho}\left[\xi\left(s_{3}\right)\right]\right] .
\end{aligned}
$$


Now, expanding this expression for $\nabla_{\mu}\left[\xi\left(s_{1}\right)\right]^{*}\left[\nabla_{\nu}\left[\xi\left(s_{2}\right)\right], \nabla_{\rho}\left[\xi\left(s_{3}\right)\right]\right]$, we get

$$
\begin{aligned}
& \nabla_{\mu}\left[\xi\left(s_{1}\right)\right]^{*}\left[\nabla_{\nu}\left[\xi\left(s_{2}\right)\right], \nabla_{\rho}\left[\xi\left(s_{3}\right)\right]\right] \\
= & \left(\frac{\delta}{\delta \xi^{\rho}\left(s_{3}\right)}+i F_{\rho}\left[\xi \mid s_{3}\right]\right)\left(\frac{\delta}{\delta \xi^{\mu}\left(s_{1}\right)}+i F_{\mu}\left[\xi \mid s_{1}\right]\right)\left(\frac{\delta}{\delta \xi^{\nu}\left(s_{2}\right)}+i F_{\nu}\left[\xi \mid s_{2}\right]\right) \\
& -\left(\frac{\delta}{\delta \xi^{\rho}\left(s_{3}\right)}+i F_{\rho}\left[\xi \mid s_{3}\right]\right)\left(\frac{\delta}{\delta \xi^{\nu}\left(s_{2}\right)}+i F_{\nu}\left[\xi \mid s_{2}\right]\right)\left(\frac{\delta}{\delta \xi^{\mu}\left(s_{1}\right)}+i F_{\mu}\left[\xi \mid s_{1}\right]\right) \\
& +\left(\frac{\delta}{\delta \xi^{\nu}\left(s_{2}\right)}+i F_{\nu}\left[\xi \mid s_{2}\right]\right)\left(\frac{\delta}{\delta \xi^{\rho}\left(s_{3}\right)}+i F_{\rho}\left[\xi \mid s_{3}\right]\right)\left(\frac{\delta}{\delta \xi^{\mu}\left(s_{1}\right)}+i F_{\mu}\left[\xi \mid s_{1}\right]\right) \\
& -\left(\frac{\delta}{\delta \xi^{\nu}\left(s_{2}\right)}+i F_{\nu}\left[\xi \mid s_{2}\right]\right)\left(\frac{\delta}{\delta \xi^{\mu}\left(s_{1}\right)}+i F_{\mu}\left[\xi \mid s_{1}\right]\right)\left(\frac{\delta}{\delta \xi^{\rho}\left(s_{3}\right)}+i F_{\rho}\left[\xi \mid s_{3}\right]\right) \\
& +\left(\frac{\delta}{\delta \xi^{\mu}\left(s_{1}\right)}+i F_{\mu}\left[\xi \mid s_{1}\right]\right)\left(\frac{\delta}{\delta \xi^{\nu}\left(s_{2}\right)}+i F_{\nu}\left[\xi \mid s_{2}\right]\right)\left(\frac{\delta}{\delta \xi^{\rho}\left(s_{3}\right)}+i F_{\rho}\left[\xi \mid s_{3}\right]\right) \\
& -\left(\frac{\delta}{\delta \xi^{\mu}\left(s_{1}\right)}+i F_{\mu}\left[\xi \mid s_{1}\right]\right)\left(\frac{\delta}{\delta \xi^{\rho}\left(s_{3}\right)}+i F_{\rho}\left[\xi \mid s_{3}\right]\right)\left(\frac{\delta}{\delta \xi^{\nu}\left(s_{2}\right)}+i F_{\nu}\left[\xi \mid s_{2}\right]\right) \\
= & 0,
\end{aligned}
$$

where

$$
\begin{aligned}
& \frac{\delta}{\delta \xi^{\mu}\left(s_{1}\right)}+i F_{\mu}\left[\xi \mid s_{1}\right] \\
= & \left(\begin{array}{cc}
\frac{\delta}{\delta \xi^{\mu}\left(s_{1}\right)}+i F_{\mu}\left[\xi \mid s_{1}\right]_{11} & i F_{\mu}\left[\xi \mid s_{1}\right]_{12} \\
i F_{\mu}\left[\xi \mid s_{1}\right]_{21} & \frac{\delta}{\delta \xi^{\mu}\left(s_{1}\right)}+i F_{\mu}\left[\xi \mid s_{1}\right]_{22}
\end{array}\right), \\
& \frac{\delta}{\delta \xi^{\nu}\left(s_{2}\right)}+i F_{\nu}\left[\xi \mid s_{2}\right] \\
= & \left(\begin{array}{cc}
\frac{\delta}{\delta \xi^{\nu}\left(s_{2}\right)}+i F_{\nu}\left[\xi \mid s_{2}\right]_{11} & i F_{\nu}\left[\xi \mid s_{2}\right]_{12} \\
i F_{\nu}\left[\xi \mid s_{2}\right]_{21} & \frac{\delta}{\delta \xi^{\nu}\left(s_{2}\right)}+i F_{\nu}\left[\xi \mid s_{2}\right]_{22}
\end{array}\right), \\
& \frac{\delta}{\delta \xi^{\rho}\left(s_{3}\right)}+i F_{\rho}\left[\xi \mid s_{3}\right] \\
= & \left(\begin{array}{cc}
\frac{\delta}{\delta \xi^{\rho}\left(s_{3}\right)}+i F_{\rho}\left[\xi \mid s_{3}\right]_{11} & i F_{\rho}\left[\xi \mid s_{3}\right]_{12} \\
i F_{\rho}\left[\xi \mid s_{3}\right]_{21} & \frac{\delta}{\delta \xi^{\rho}\left(s_{3}\right)}+i F_{\rho}\left[\xi \mid s_{3}\right]_{22}
\end{array}\right) .
\end{aligned}
$$

Thus, we get, $\nabla_{\mu}\left[\xi\left(s_{1}\right)\right]^{*}\left[\nabla_{\nu}\left[\xi\left(s_{2}\right)\right], \nabla_{\rho}\left[\xi\left(s_{3}\right)\right]\right]=0$.

However, as the curvature of the loop space is generated by the commutator of the functional covariant derivatives, we observe that the functional Bianchi identity is satisfied for the loop space. So, we can write $\nabla_{\rho}\left[\xi\left(s_{3}\right)\right] G_{\mu \nu}\left[\xi\left(s_{1}, s_{2}\right)\right]+$ $\nabla_{\mu}\left[\xi\left(s_{1}\right)\right] G_{\nu \rho}\left[\xi\left(s_{2}, s_{3}\right)\right]+\nabla_{\nu}\left[\xi\left(s_{2}\right)\right] G_{\rho \mu}\left[\xi\left(s_{3}, s_{1}\right)\right]=0$. We emphasize again that in the presence of a monopole, the space-time Bianchi identity is not satisfied, and this translates into the non-vanishing of the loop space curvature $G_{\mu \nu}\left[\xi\left(s_{1}, s_{2}\right)\right]$. On the other hand, the loop space curvature itself does satisfy the Bianchi identity even in the presence of a monopole.

\section{Loop of Loops}

In the previous section we analysed Polyakov loops for the ABJ theory. It may be noted that Polyakov loops have been generalized to loop of loops for Yang- 
Mills theories 41. Here we will apply this formalism of loop of loops to the ABJ theory. We will also extend this formalism to include the concept of a connection and curvature for loop of loops. A loop in the loop space can be defined using $F_{\mu}[\xi \mid s]$ as the connection. Now we can parameterize a loop in the loop space as follows [41,

$$
\Sigma:\left\{\xi^{\mu}(t: s), s=0 \rightarrow 2 \pi, t=0 \rightarrow 2 \pi\right\},
$$

where $\xi^{\mu}(t: 0)=\xi^{\mu}(t: 2 \pi)$, and $t=0 \rightarrow 2 \pi$. At each value of $t$, a closed loop $C(t)$ is traced in the spacetime passing through a fixed point. Thus, for $t=0$ and $t=2 \pi$ it shrinks to this fixed point and as $t$ varies from $0 \rightarrow 2 \pi, C(t)$ traces out a closed loop in the loop space. This loop starts and ends at the fixed point to which $C(t)$ shrinks for $t=0$ and $t=2 \pi$. Thus, we can define a loop variable for this space as,

$$
\begin{aligned}
\Theta[\xi]= & \left(\begin{array}{ll}
\Theta_{11}[\xi] & \Theta_{12}[\xi] \\
\Theta_{21}[\xi] & \Theta_{22}[\xi]
\end{array}\right) \\
= & P_{t} \exp i \int_{0}^{2 \pi} d t \int_{0}^{2 \pi} d s\left(\begin{array}{cc}
F^{\mu}[\xi \mid t: s]_{11} & F^{\mu}[\xi \mid t: s]_{12} \\
F^{\mu}[\xi \mid t: s]_{21} & F^{\mu}[\xi \mid t: s]_{22}
\end{array}\right) \\
& \times \frac{\partial \xi_{\mu}(t: s)}{\partial t},
\end{aligned}
$$

where $P_{t}$ denotes ordering in $t$ increasing from right to left and the derivative is taken from below. The connection in the loop space, $F^{\mu}[\xi \mid s]$, plays the role of the space-time gauge field $\mathcal{A}[\xi]$ in the loop space, so that this definition is the analogue in loop space of $(5)$. However, the connection in the loop space is infinite dimensional and so apart from the sum over $\mu$, we have to also integrate over $s$. In ordinary spacetime this parametrized loop in loop space is represented by a two dimensional surface which enclosing a three dimensional volume.

In analogy with the previous case we can define a connection in this space using $\Theta[\xi]$. In fact, we will define the connection in this space to be the logarithmic derivative of $\Theta[\xi]$. So, we write

$$
B_{\mu}[\xi \mid t: s]=\left(\begin{array}{ll}
B_{\mu}[\xi \mid t: s]_{11} & B_{\mu}[\xi \mid t: s]_{12} \\
B_{\mu}[\xi \mid t: s]_{21} & B_{\mu}[\xi \mid t: s]_{22}
\end{array}\right),
$$

where

$$
B_{\mu}[\xi \mid t: s]=i \Theta^{-1}[\xi] \frac{\delta}{\delta \xi^{\mu}(t: s)} \Theta[\xi] .
$$

Geometrically, $B_{\mu}[\xi \mid t: s]$ can be regarded as a connection in the space of loop of loops, as it represents the change in $\Theta[\xi]$ as one moves from one point in this space to its neighboring point. Now we can define a quantity which will act as parallel transport in this space

$$
\begin{aligned}
\Theta\left[\xi\left(t_{1}, t_{2}\right)\right]= & \left(\begin{array}{cc}
\Theta_{11}\left[\xi\left(t_{1}, t_{2}\right)\right] & \Theta_{12}\left[\xi\left(t_{1}, t_{2}\right)\right] \\
\Theta_{21}\left[\xi\left(t_{1}, t_{2}\right)\right] & \Theta_{22}\left[\xi\left(t_{1}, t_{2}\right)\right]
\end{array}\right) \\
= & P_{t} \exp i \int_{t_{1}}^{t_{2}} d t \int_{0}^{2 \pi} d s\left(\begin{array}{cc}
F^{\mu}[\xi \mid t: s]_{11} & F^{\mu}[\xi \mid t: s]_{12} \\
F^{\mu}[\xi \mid t: s]_{21} & F^{\mu}[\xi \mid t: s]_{22}
\end{array}\right) \\
& \times \frac{\partial \xi_{\mu}(t: s)}{\partial t} .
\end{aligned}
$$


Now using $\Theta\left[\xi\left(t_{1}, t_{2}\right)\right]$, we can move from a fixed point another point and then take a detour and travel backwards along the same path to then original path. In doing this the phase factor for the generated in going from the original point to final point, exactly cancels the phase factor generated in going back from the final point to the original point. However, the phase factor while transporting around the infinitesimal circuit at the final point does have a finite contribution that does not cancel. This contribution is proportional to the curvature of the loop space. In fact, by repeating the previous calculations, we observe that

$$
\begin{aligned}
B_{\mu}\left[\xi\left(t_{1}: s_{1}\right)\right]= & \int d s_{2} \Theta^{-1}\left[\xi\left(t_{1}, 0\right)\right] G_{\mu \nu}\left[\xi\left(t_{1}: s_{1}, s_{2}\right)\right] \Theta\left[\xi\left(0, t_{1}\right)\right] \\
& \times \frac{\partial \xi^{\nu}\left(t_{1}: s_{2}\right)}{\partial t_{1}}
\end{aligned}
$$

So, $B^{\mu}\left[\xi\left(t_{1}: s_{1}\right)\right]$ is proportional to the curvature of the loop space. Now for $s_{1} \neq s_{2}, G_{\mu \nu}\left[\xi\left(t_{1}: s_{1}, s_{2}\right)\right]$ corresponds to a parameterized surface enclosing no volume and $B^{\mu}\left[\xi\left(t_{1}: s_{1}\right)\right]$ in this case is zero. The same value is obtained for $s_{1}=s_{2}$, if it the volume $\Sigma$ encloses does not contain a monopole.

As in the space of loop of loops $B^{\mu}[\xi(t: s)]$ acts like a connection, we can construct a covariant derivative using it,

$$
\bar{\nabla}_{\mu}[\xi(t: s)]=\frac{\delta}{\delta \xi^{\mu}(t: s)}+i B_{\mu}[\xi \mid t: s]
$$

We can now define a curvature $-i E_{\mu \nu}\left[\xi\left(t_{1}, t_{2}: s_{1}, s_{2}\right)\right]$ of this space as follows $\left[\bar{\nabla}_{\mu}\left[\xi\left(t_{1}: s_{1}\right)\right], \bar{\nabla}_{\nu}\left[\xi\left(t_{2}: s_{2}\right)\right]\right]$. Thus, we can write

$$
\begin{aligned}
E_{\mu \nu}\left[\xi\left(t_{1}, t_{2}: s_{1}, s_{2}\right)\right]= & \frac{\delta}{\delta \xi^{\mu}\left(t_{2}: s_{2}\right)} B_{\nu}\left[\xi \mid t_{1}: s_{1}\right]-\frac{\delta}{\delta \xi^{\nu}\left(t_{1}: s_{1}\right)} B_{\mu}\left[\xi \mid t_{2}: s_{2}\right] \\
& +i\left[B_{\mu}\left[\xi \mid t_{1}: s_{1}\right], B_{\mu}\left[\xi \mid t_{2}: s_{2}\right]\right] .
\end{aligned}
$$

The gauge transformations in loop space can be denoted by given by $v=$ $\exp i \Lambda[\xi]$. The connection $B_{\mu}[\xi \mid t: s]$ transforms under these gauge transformations as $B_{\mu}[\xi \mid t: s]=i v \bar{\nabla}_{\mu}[\xi(t: s)] v^{-1}$ and $E_{\mu \nu}\left[\xi\left(t_{1}, t_{2}: s_{1}, s_{2}\right)\right]$ transforms under these gauge transformations as $v E_{\mu \nu}\left[\xi\left(t_{1}, t_{2}: s_{1}, s_{2}\right)\right] v^{-1}$.

It may be noted that $E_{\mu \nu}\left[\xi\left(t_{1}, t_{2}: s_{1}, s_{2}\right)\right]$ again satisfies a functional Bianchi identity. Now we define $\bar{\nabla}_{\mu}\left[\xi\left(t_{1}: s_{1}\right)\right]^{*}\left[\bar{\nabla}_{\nu}\left[\xi\left(t_{2}: s_{2}\right)\right], \bar{\nabla}_{\rho}\left[\xi\left(t_{3}, s_{3}\right)\right]\right]$ as follows,

$$
\begin{aligned}
& \bar{\nabla}_{\mu}\left[\xi\left(t_{1}: s_{1}\right)\right]^{*}\left[\bar{\nabla}_{\nu}\left[\xi\left(t_{2}: s_{2}\right)\right], \bar{\nabla}_{\rho}\left[\xi\left(t_{3}: s_{3}\right)\right]\right] \\
= & \bar{\nabla}_{\rho}\left[\xi\left(t_{3}: s_{3}\right)\right]\left[\bar{\nabla}_{\mu}\left[\xi\left(t_{1}: s_{1}\right)\right], \bar{\nabla}_{\nu}\left[\xi\left(t_{2}: s_{2}\right)\right]\right] \\
& +\bar{\nabla}_{\nu}\left[\xi\left(t_{2}: s_{2}\right)\right]\left[\bar{\nabla}_{\rho}\left[\xi\left(t_{3}: s_{3}\right)\right], \bar{\nabla}_{\mu}\left[\xi\left(t_{1}: s_{1}\right)\right]\right] \\
& +\bar{\nabla}_{\mu}\left[\xi\left(t_{1}: s_{1}\right)\right]\left[\bar{\nabla}_{\nu}\left[\xi\left(t_{2}: s_{2}\right)\right], \bar{\nabla}_{\rho}\left[\xi\left(t_{3}: s_{3}\right)\right]\right] .
\end{aligned}
$$

In order to prove the functional Bianchi identity for $E_{\mu \nu}\left[\xi\left(t_{1}, t_{2}: s_{1}, s_{2}\right)\right]$, we 
expand $\bar{\nabla}_{\mu}\left[\xi\left(t_{1}: s_{1}\right)\right]^{*}\left[\bar{\nabla}_{\nu}\left[\xi\left(t_{2}: s_{2}\right)\right], \bar{\nabla}_{\rho}\left[\xi\left(t_{3}: s_{3}\right)\right]\right]$, as follows

$$
\begin{aligned}
& \bar{\nabla}_{\mu}\left[\xi\left(t_{1}: s_{1}\right)\right]^{*}\left[\bar{\nabla}_{\nu}\left[\xi\left(t_{2}: s_{2}\right)\right], \bar{\nabla}_{\rho}\left[\xi\left(t_{3}: s_{3}\right)\right]\right] \\
= & \left(\frac{\delta}{\delta \xi^{\rho}\left(t_{3}: s_{3}\right)}+i B_{\rho}\left[\xi \mid t_{3}: s_{3}\right]\right)\left(\frac{\delta}{\delta \xi^{\mu}\left(t_{1}: s_{1}\right)}+i B_{\mu}\left[\xi \mid t_{1}: s_{1}\right]\right) \\
& \times\left(\frac{\delta}{\delta \xi^{\nu}\left(t_{2}: s_{2}\right)}+i B_{\nu}\left[\xi \mid t_{2}: s_{2}\right]\right) \\
& \times\left(\frac{\delta}{\delta \xi^{\rho}\left(t_{3}: s_{3}\right)}+i B_{\rho}\left[\xi \mid t_{3}: s_{3}\right]\right)\left(\frac{\delta}{\delta \xi^{\mu}\left(t_{1}: s_{1}\right)}+i B_{\mu}\left[\xi \mid t_{1}: s_{1}\right]\right) \\
& +\left(\frac{\delta}{\delta \xi^{\nu}\left(t_{2}: s_{2}\right)}+i B_{\nu}\left[\xi \mid t_{2}: s_{2}\right]\right) \\
& \times\left(\frac{\delta}{\delta \xi^{\mu}\left(t_{1}: t_{1}\right)}+i s_{\mu}\left[\xi \mid t_{1}: s_{1}\right]\right)\left(\frac{\delta}{\delta \xi^{\rho}\left(t_{3}: s_{3}\right)}+i B_{\rho}\left[\xi \mid t_{3}: s_{3}\right]\right) \\
& -\left(\frac{\delta}{\delta \xi^{\nu}\left(t_{2}: s_{2}\right)}+i B_{\nu}\left[\xi \mid t_{2}: s_{2}\right]\right)\left(\frac{\delta}{\delta \xi^{\mu}\left(t_{1}: s_{1}\right)}+i B_{\mu}\left[\xi \mid t_{1}: s_{1}\right]\right) \\
& \times\left(\frac{\delta}{\delta \xi^{\rho}\left(t_{3}: s_{3}\right)}+i B_{\rho}\left[\xi \mid t_{3}: s_{3}\right]\right) \\
& +\left(\frac{\delta}{\delta \xi^{\mu}\left(t_{1}: s_{1}\right)}+i B_{\mu}\left[\xi \mid t_{1}: s_{1}\right]\right)\left(\frac{\delta}{\delta \xi^{\nu}\left(t_{2}: s_{2}\right)}+i B_{\nu}\left[\xi \mid t_{2}: s_{2}\right]\right) \\
& \times\left(\frac{\delta}{\delta \xi^{\rho}\left(t_{3}: s_{3}\right)}+i B_{\rho}\left[\xi \mid t_{3}: s_{3}\right]\right) \\
& -\left(\frac{\delta}{\delta \xi^{\mu}\left(t_{1}: s_{1}\right)}+i B_{\mu}\left[\xi \mid t_{1}: s_{1}\right]\right)\left(\frac{\delta}{\delta \xi^{\rho}\left(t_{3}: s_{3}\right)}+i B_{\rho}\left[\xi \mid t_{3}: s_{3}\right]\right) \\
& \times\left(\frac{\delta}{\delta \xi^{\nu}\left(t_{2}: s_{2}\right)}+i B_{\nu}\left[\xi \mid t_{2}: s_{2}\right]\right) \\
& 0
\end{aligned}
$$

where

$$
\begin{aligned}
& \frac{\delta}{\delta \xi^{\mu}\left(t_{1}: s_{1}\right)}+i B_{\mu}\left[\xi \mid t_{1}: s_{1}\right] \\
= & \left(\begin{array}{cc}
\frac{\delta}{\delta \xi^{\mu}\left(t_{1}: s_{1}\right)}+i B_{\mu}\left[\xi \mid t_{1}: s_{1}\right]_{11} & i B_{\mu}\left[\xi \mid t_{1}: s_{1}\right]_{12} \\
i B_{\mu}\left[\xi \mid t_{1}: s_{1}\right]_{21} & \frac{\delta}{\delta \xi^{\mu}\left(t_{1}: s_{1}\right)}+i B_{\mu}\left[\xi \mid t_{1}: s_{1}\right]_{22}
\end{array}\right), \\
& \frac{\delta}{\delta \xi^{\nu}\left(t_{2}: s_{2}\right)}+i B_{\nu}\left[\xi \mid t_{2}: s_{2}\right] \\
= & \left(\begin{array}{cc}
\frac{\delta}{\delta \xi^{\nu}\left(t_{2}: s_{2}\right)}+i B_{\nu}\left[\xi \mid t_{2}: s_{2}\right]_{11} & i B_{\nu}\left[\xi \mid t_{2}: s_{2}\right]_{12} \\
i B_{\nu}\left[\xi \mid t_{2}: s_{2}\right]_{21} & \frac{\delta}{\delta \xi^{\nu}\left(t_{2}: s_{2}\right)}+i B_{\nu}\left[\xi \mid t_{2}: s_{2}\right]_{22}
\end{array}\right), \\
& \frac{\delta}{\delta \xi^{\rho}\left(t_{3}: s_{3}\right)}+i B_{\rho}\left[\xi \mid t_{3}: s_{3}\right] \\
= & \left(\begin{array}{cc}
\frac{\delta}{\delta \xi^{\rho}\left(t_{3}: s_{3}\right)}+i B_{\rho}\left[\xi \mid t_{3}: s_{3}\right]_{11} & i B_{\rho}\left[\xi \mid t_{3}: s_{3}\right]_{12} \\
i B_{\rho}\left[\xi \mid t_{3}: s_{3}\right]_{21} & \frac{\delta}{\delta \xi^{\rho}\left(t_{3}: s_{3}\right)}+i B_{\rho}\left[\xi \mid t_{3}: s_{3}\right]_{22}
\end{array}\right) .
\end{aligned}
$$

Thus, we get, $\bar{\nabla}_{\mu}\left[\xi\left(t_{1}: s_{1}\right)\right]^{*}\left[\bar{\nabla}_{\nu}\left[\xi\left(t_{2}: s_{2}\right)\right], \bar{\nabla}_{\rho}\left[\xi\left(t_{3}: s_{3}\right)\right]\right]=0$. 
However, as the curvature of the loop space is generated by the commutator of the functional covariant derivatives, we observe that the functional Bianchi identity is satisfied for this space. So, we can write $\bar{\nabla}_{\rho}\left[\xi\left(t_{3}: s_{3}\right)\right] E_{\mu \nu}\left[\xi\left(t_{1}, t_{2}\right.\right.$ : $\left.\left.s_{1}, s_{2}\right)\right]+\bar{\nabla}_{\mu}\left[\xi\left(t_{1}: s_{1}\right)\right] E_{\nu \rho}\left[\xi\left(t_{2}, t_{3}: s_{2}, s_{3}\right)\right]+\bar{\nabla}_{\nu}\left[\xi\left(t_{2}: s_{2}\right)\right] E_{\rho \mu}\left[\xi\left(t_{3}, t_{1}: s_{3}, s_{1}\right)\right]=$ 0 .

\section{Topological Defects}

The both the ABJM theory and the ABJ theory have $\mathcal{N}=6$ supersymmetry. However, it is expected that for ABJM theory for the Chern-Simons levels, $k=1,2$, this supersymmetry will get enhanced to $\mathcal{N}=8$ supersymmetry 10 , 11, 12, 13. In this supersymmetric enhancement an important role is played by the monopole operators. Thus, it is important to understand the role of monopoles in the ABJM theory. In fact, in this section we will analyse the monopoles in the ABJ theory. We will also study a topological defect in the loop space. This defect in loop space is similar to a monopole in spacetime.

So, now we will analyse monopoles in the ABJ theory. To do that, we first note that whenever $\mathcal{F}$ is derivable from the superconnection $\mathcal{A}$, Bianchi identities for $\mathcal{F}$ will be satisfied, $\nabla^{*} \mathcal{F}=0$. As the curvature of the loop space is proportional to the Bianchi identities, it will vanish whenever $\mathcal{F}$ is derivable from the superconnection $\mathcal{A}$. However, at a point where the loop intersects the world-line of a monopole, $\mathcal{F}$ will not be derivable from the superconnection $\mathcal{A}$ and the Bianchi identities will not hold. Thus, the above argument will not hold and the curvature can get a non-zero value. In other words if $G_{A B}\left[\xi\left(s_{1}, s_{2}\right)\right] \neq 0$ then $\nabla^{*} \mathcal{F} \neq 0$ and the loop will be intersecting word-lines of a monopole.

As $\Theta$ measure the total change in the loop as $t=0 \rightarrow 2 \pi$, so, if a monopole is present it will not wind fully around the gauge group. However, in absence of a monopole, it will wind fully around the gauge group. Thus, we can write [41] $\Theta=\zeta I$, where $\zeta$ is the monopole charge of the ABJ theory enclosed by the surface $\Sigma$. Thus, the monopole charge corresponds to the loop in the loop space for the ABJ theory. If the loop passes through a monopole, then at the value of $s_{1}$, where the loop $\xi\left(s_{1}\right)$ intersects the monopole world-line $Y\left(s_{3}\right)$, the curvature will not vanish. In fact, it will be given by [41]

$$
\begin{aligned}
G_{\mu \nu}\left[\xi\left(s_{1}, s_{2}\right)\right]= & -\pi \int d s_{3} \kappa[\xi \mid s] \epsilon_{\mu \nu \rho \tau} \frac{d \xi^{\rho}\left(s_{1}\right)}{d s_{1}} \frac{d \xi^{\tau}\left(s_{3}\right)}{d s_{3}} \\
& \times \delta^{3}\left(\xi\left(s_{1}\right)-Y\left(s_{3}\right)\right) \delta\left(s_{1}-s_{2}\right)
\end{aligned}
$$

Here $\kappa[\xi \mid s]$ satisfies $\exp i \pi \kappa=\zeta$, where, $\zeta$ is the charge carried by the monopole moving along the world-line $Y\left(s_{3}\right)$.

We have observed that even when monopoles are present a functional Bianchi identity for the curvature in the loop space is satisfied. Furthermore, for the space of loop of loops, let us first travel from point say $t_{1}$ along a certain direction till a point say $t_{2}$. After that we travel along another direction at $t_{1}$, then we travel along the first direction and finally again travel along the direction we traveled from $t_{2}$, to get to $t_{1}$. In doing so we completed a full circuit and the total change in phase generated in the process is represented is proportional to the curvature $-i E_{\mu \nu}\left[\xi\left(t_{1}, t_{2}: s_{1}, s_{2}\right)\right] \delta \xi^{\mu}\left(t_{1}: s_{1}\right) \xi^{\nu}\left(t_{2}: s_{2}\right)$. Now we calculate the quantity given by $\Theta^{-1}\left[\xi_{2}\right] \Theta\left[\xi_{3}\right]-\Theta[\xi] \Theta\left[\xi_{1}\right]$, where $\xi_{1}^{\mu}[t: s]=\xi^{\mu}[t: s]+\delta \xi^{\mu}[t$ : 
$s], \xi_{2}^{\mu}[t: s]=\xi^{\mu}[t: s]+\delta^{\prime} \xi^{\mu}[t: s], \xi_{3}^{\mu}[t: s]=\xi_{1}^{\mu}[t: s]+\delta^{\prime} \xi^{\mu}[t: s]$. Now we can write

$$
\begin{aligned}
\Theta\left[\xi_{1}\right]= & \Theta[\xi]-i g \int d t \int d s_{1} d s_{2} \Theta[\xi(2 \pi, t)] G_{\mu \nu}\left[\xi\left(t: s_{1}, s_{2}\right)\right] \\
& \times \frac{\partial \xi^{\nu}\left(t: s_{2}\right)}{\partial t} \delta \xi^{\mu}\left(t, s_{1}\right) \Theta[\xi(t, 0)] .
\end{aligned}
$$

We also have

$$
\begin{aligned}
\Theta\left[\xi_{2}\right]= & \Theta[\xi]-i g \int d t \int d s_{1} d s_{2} \Theta[\xi(2 \pi, t)] G_{\mu \nu}\left[\xi\left(t: s_{1}, s_{2}\right)\right] \\
& \times \frac{\partial \xi^{\nu}\left(t: s_{2}\right)}{\partial t} \delta^{\prime} \xi^{\mu}\left(t, s_{1}\right) \Theta[\xi(t, 0)] .
\end{aligned}
$$

Finally, we have

$$
\begin{aligned}
\Theta\left[\xi_{3}\right]= & \Theta\left[\xi_{1}\right]-i g \int d t \int d s_{1} d s_{2} \Theta\left[\xi_{1}(2 \pi, t)\right] G_{\mu \nu}\left[\xi_{1}\left(t: s_{1}, s_{2}\right)\right] \\
& \times \frac{\partial \xi_{1}^{\nu}\left(t: s_{2}\right)}{\partial t} \delta^{\prime} \xi_{1}^{\mu}\left(t, s_{1}\right) \Theta\left[\xi_{1}(t, 0)\right] .
\end{aligned}
$$

We also note that

$$
\begin{aligned}
G_{\mu \nu}\left[\xi_{1}\left(t: s_{1}, s_{2}\right)\right]= & \int d s_{3} \frac{\delta}{\delta \xi^{\rho}\left(t: s_{3}\right)} G_{\mu \nu}\left[\xi\left(t: s_{1}, s_{2}\right)\right] \delta \xi^{\rho}\left(t: s_{3}\right) \\
& +G_{\mu \nu}\left[\xi\left(t: s_{1}, s_{2}\right)\right] .
\end{aligned}
$$

Now by collection all the terms for $E_{\mu \nu}\left[\xi\left(t_{1}, t_{2}: s_{1}, s_{2}\right)\right]$ is given by

$$
\begin{aligned}
E_{\mu \nu}\left[\xi\left(t_{1}, t_{2}: s_{1}, s_{2}\right)\right]= & \int d s_{3} \Theta^{-1}\left[\xi\left(t_{1}, 0\right)\right]\left[\nabla_{\rho}\left[\xi\left(t_{1}: s_{3}\right)\right] G_{\mu \nu}\left[\xi\left(t_{1}: s_{1}, s_{2}\right)\right]\right. \\
& +\nabla_{\mu}\left[\xi\left(t_{1}: s_{1}\right)\right] G_{\nu \rho}\left[\xi\left(t_{1}: s_{2}, s_{3}\right)\right] \\
& \left.+\nabla_{\nu}\left[\xi\left(t_{1}: s_{2}\right)\right] G_{\rho \mu}\left[\xi\left(t_{1}: s_{3}, s_{1}\right)\right]\right] \\
& \times \Theta\left[\xi\left(t_{1}, 0\right)\right] \frac{\partial \xi^{\rho}\left(t_{1}: s_{3}\right)}{\partial t_{1}} \delta\left(t_{1}-t_{2}\right) .
\end{aligned}
$$

So, the curvature of the space of loop of loops is proportional to the functional Bianchi identity, $\nabla_{\rho}\left[\xi\left(t_{1}: s_{3}\right)\right] G_{\mu \nu}\left[\xi\left(t_{1}: s_{1}, s_{2}\right)\right]+\nabla_{\mu}\left[\xi\left(t_{1}: s_{1}\right)\right] G_{\nu \rho}\left[\xi\left(t_{1}:\right.\right.$ $\left.\left.s_{2}, s_{3}\right)\right]+\nabla_{\nu}\left[\xi\left(t_{1}: s_{2}\right)\right] G_{\rho \mu}\left[\xi\left(t_{1}: s_{3}, s_{1}\right)\right]$.

If this functional Bianchi identity is satisfied, then this curvature vanishes. However, one can envisage a singularity in loop space which is similar to the singularity in space-time giving a monopole. If such a monopole like defect occurs, such that the functional Bianchi identity is not satisfied, then the curvature for loop of loops will not be zero. It would be interesting to see what would be the possible implications of such a topological defect. It may be noted that even in presence of such a defect, the functional Bianchi identity for $E_{\mu \nu}\left[\xi\left(t_{1}, t_{2}: s_{1}, s_{2}\right)\right]$ hold, $\bar{\nabla}_{\rho}\left[\xi\left(t_{3}: s_{3}\right)\right] E_{\mu \nu}\left[\xi\left(t_{1}, t_{2}: s_{1}, s_{2}\right)\right]+\bar{\nabla}_{\mu}\left[\xi\left(t_{1}:\right.\right.$ $\left.\left.s_{1}\right)\right] E_{\nu \rho}\left[\xi\left(t_{2}, t_{3}: s_{2}, s_{3}\right)\right]+\bar{\nabla}_{\nu}\left[\xi\left(t_{2}: s_{2}\right)\right] E_{\rho \mu}\left[\xi\left(t_{3}, t_{1}: s_{3}, s_{1}\right)\right]=0$.

\section{Conclusion}

In this paper we analysed Polyakov loops for the ABJ theory. This was done by first constructing the loop variables in terms of a superconnection. The fermions 
coupled to the loop in the bi-fundamental representation. Then a connection for this loop was constructed and a curvature tensor from this connection was also constructed. This curvature tensor was found to be proportional to the Bianchi identities and thus vanished when they were satisfied. As the Bianchi identities are not satisfied in presence of a monopole, so this curvature tensor has a non-vanishing value in the presence of a monopole. A space of loop of loop was also construed and was used as a measure for the monopole charge of the ABJ theory. We also constructed the curvature and connection for this space of loop of loops. Furthermore, certain topological defects in the loops space were analysed using the curvature of the space of loop of loops. It would be interesting to investigate further the physical implications of topological defect in the loop space. If a loop in the loop space is really the quantity which captures real physics, then it seems likely that a topological defect in this space of loop of loops can have real physical meaning. Furthermore, it would be interesting to see how far can we go with such constructions. We have already constructed the functional Bianchi identity for the space of loop of loops. The next natural question to analyse is the existence of topological defects in this space which violates this functional Bianchi identity. The we can perform a similar analyse for those defect too.

If we consider the ABJM theory and give a vacuum expectation value to one of the scalar fields, then we arrive at the action for multiple D2-branes [44, 45, 46, 47]. In this mechanism the gauge group $U(N) \times U(N)$ is broken down to its diagonal subgroup. The theory thus obtained is the Yang-Mills theory coupled to matter fields. It would be interesting to analyse the Polyakov loops for the ABJM theory, after a vacuum expectation value is given to one of the scalar fields. It would be expected that the Polyakov loops for the ABJM theory in this case will reduce to the Polyakov loop for the D2-branes. Furthermore, it will interesting to analyse what happens to monopole charge of the ABJM theory, after a vacuum expectation value is given to one of the scalar fields. The Wilsons loops in generic tensor representation for IIB string theory are dual to D3-branes [48].

In fact, they are also dual to D5-branes [49. It is thus expected that string like objects in $A d S_{3} \times C P^{3}$ will be dual to Wilsons loops in different representation of $S U(N \mid M)$ 50. It would be interesting to construct such operators for the ABJM theory and use them for perturbative calculations. It will also be interesting to analyse what happens to this duality when the Wilsons loops are replaced by Polyakov loops.

A system of M2-branes ending on other objects in M-theory has been studied by analysing the ABJM theory and the BLG theory on a manifold with boundaries [51, 52, 53. Boundary conditions for the M2-branes ending on M5branes, M9-branes and gravitational waves have been studied 54. Furthermore, a background flux can exist in M-theory. Boundary conditions for M2-branes in the presence of a background flux have also been discussed [55. It is possible to learn about the physics of M5-branes by studding a system of M2-branes ending on them. A novel quantum geometry on M5-branes has been studied by analysing a system of M2-branes ending on a M5-brane with constant $C$-field [56]. The BLG theory was used to study this novel geometry. In fact, the BLG theory with Nambu-Poisson 3-bracket has been identified with the M5-brane action with a large worldvolume C-field [57].

A non-commutative string theory on the M5-brane worldvolume has been 
derived using the action of a single M2-brane [58, 59, 60. It would be interesting to analyse the boundary effects for fractional M2-branes using ABJ theory. It would also be interesting to analyse topological defects in this theory using Polyakov loops.

\section{References}

[1] A. Gustavsson, JHEP. 0804, 083 (2008)

[2] J. Bagger and N. Lambert, JHEP. 0802, 105 (2008)

[3] J. Bagger and N. Lambert, Phys. Rev. D77, 065008 (2008)

[4] M. A. Bandres, A. E. Lipstein and J. H. Schwarz, JHEP. 0809, 027 (2008)

[5] E. Antonyan and A. A. Tseytlin, Phys. Rev. D79, 046002 (2009)

[6] O. Aharony, O. Bergman, D. L. Jafferis and J. Maldacena, JHEP. 0810, $091(2008)$

[7] M. A. Bandres, A. E. Lipstein and J. H. Schwarz, JHEP. 0809, 027 (2008)

[8] M. Schnabl and Y. Tachikawa, JHEP. 1009, 103 (2010)

[9] A. Mauri, A. Santambrogio and S. Scoleri, JHEP. 04, 146 (2013)

[10] O. K. Kwon, P. Oh and J. Sohn, JHEP. 0908, 093 (2009)

[11] I. R. Klebanov and G. Torri, Int. J. Mod. Phys. A25, 332 (2010)

[12] O. K. Kwon, P. Oh, C. Sochichiu and J. Sohn, JHEP. 1003, 092 (2010)

[13] H. Samtleben and R. Wimmer, JHEP. 1010, 080 (2010)

[14] O. Aharony, O. Bergman and D. L. Jafferis, JHEP. 0811, 043 (2008)

[15] S. Cremonesi, JHEP. 1101, 076 (2011)

[16] J. Evslin and S. Kuperstein, JHEP. 0912, 016 (2009)

[17] J. A. Minahan, O. Ohlsson Sax and C. Sieg, J. Phys. A43, 275402 (2010)

[18] P. Caputa, C. Kristjansen and K. Zoubos, Phys. Lett. B 677, 197 (2009)

[19] O. Aharony, O. Bergman, D. L. Jafferis and J. Maldacena, JHEP 0810, 091 (2008)

[20] O. Aharony, O. Bergman and D. L. Jafferis, JHEP. 0811, 043 (2008)

[21] W. J. Rey and J. T. Yee, Eur. Phys. J. C 22, 379 (2001)

[22] J. M. Maldacena, Phys. Rev. Lett. 80, 4859 (1998)

[23] N. Drukker and D. Trancanelli, JHEP. 1002, 058 (2010)

[24] N. Drukker, J. Plefka and D. Young, JHEP. 0811, 019 (2008)

[25] S. J. Rey, T. Suyama and S. Yamaguchi, JHEP. 0903, 127 (2009) 
[26] A. Kapustin, B. Willett and I. Yaakov, JHEP. 1003, 089 (2010)

[27] A. M. Polyakov, Nucl. Phys. 164, 171 (1980)

[28] H. M. Chan, J. Faridani and S. T. Tsou, Phys. Rev. D52, 6134 (1995)

[29] H. M. Chan, J. Faridani and S. T. Tsou, Phys. Rev. D53 7293 (1996)

[30] H. M. Chan and S. T. Tsou, Phys. Rev. D56, 3646 (1997)

[31] H. M. Chan J. Bordes, and S. T. Tsou, Int. Jour. Mod. Phys. A14, 2173 (1999)

[32] H. M. Chan and S. T. Tsou, Acta. Phys. Polon. B28, 3027 (1997)

[33] H. M. Chan and S. T. Tsou, Acta. Phys. Polon. B33, 4041 (2002)

[34] H. M. Chan, Int. J. Mod. Phys. A16, 163 (2001)

[35] H. M. Chan and S. T. Tsou, Phys. Rev. D57, 2507 (1998)

[36] H. M. Chan and S. T. Tsou, Acta. Phys. Polon. B28, 3041 (1997)

[37] H. M. Chan, J. Faridani, J. Pfaudler and S. T. Tsou, Phys. Rev. D55, 5015 (1997)

[38] M. Faizal, Euro. Phys. Lett. 103, 21003 (2013)

[39] H. M. Chan, P. Scharbach and S. T. Tsou, Ann. Phys. 167454 (1986)

[40] H. M. Chan and S. T. Tsou, Act. Phys. Pol. B17, 259 (1986)

[41] H. M. Chan and S. T. Tsou, Some Elementary Gauge Theory Concepts, World Scientific, (1993)

[42] V. Cardinali, L. Griguolo, G. Martelloni and D. Seminara, arXiv:1209.4032

[43] S. T. Tsou and I. Zois, Rept. Math. Phys. 45, 229 (2000)

[44] S. Mukhi and C. Papageorgakis, JHEP. 0805, 085 (2008)

[45] T. Li, Y. Liu and D. Xie, Int. J. Mod. Phys. A24, 3039 (2009)

[46] Y. Pang and T. Wang, Phys. Rev. D78, 125007 (2008)

[47] P. M. Ho, Y. Imamura and Y. Matsuo, JHEP. 0807, 003 (2008)

[48] J. Gomis and F. Passerini, JHEP. 0608, 074 (2006)

[49] S. Yamaguchi, JHEP. 0605, 037 (2006)

[50] J. Kluson and K. L. Panigrahi, Eur. Phys. J. C67, 565 (2010)

[51] M. Faizal and D. J. Smith, Phys. Rev. D 85, 105007 (2012)

[52] D. S. Berman and D. C. Thompson, Nucl. Phys. B820, 503 (2009)

[53] M. Faizal, JHEP. 1204, 017 (2012) 
[54] D. S. Berman, M. J. Perry, E. Sezgin and D. C. Thompson, JHEP 1004, $025(2010)$

[55] C. S. Chu and G. S. Sehmbi, J. Phys. A 44, 134504 (2011)

[56] C. S. Chu and D. J. Smith, JHEP. 0904, 097 (2009)

[57] P. M. Ho, Chin. J. Phys. 48, 1 (2010)

[58] E. Bergshoeff, D. S. Berman, J. P. van der Schaar and P. Sundell, Nucl. Phys. B 590, 173 (2000)

[59] S. Kawamoto and N. Sasakura, JHEP 0007, 014 (2000)

[60] D. S. Berman and B. Pioline, Phys. Rev. D 70, 045007 (2004) 